\title{
Materials Research Society of India Hosts Fourth Annual General Meeting
}

The Thiruvananthapuram Local Chapter of MRS-I hosted the Fourth Annual General Meeting of the Materials Research Society of India (MRS-I) from February 9 to 11, 1993, at the Regional Research Laboratory, Thiruvananthapuram. The meeting was attended by about 200 participants from more than 50 organizations.

In his inaugural address, P. Rama Rao, president of MRS-I, focused on the liberalization of the Indian economy and its integration with global activity. These developments have serious implications for the development of Indian technology-which must now achieve international competitiveness-and they require intense cooperation among academia, research and development centers, and industries. As examples of successes emerging from such interactions, Rao cited the development of biomaterials and liquid crystal displays. He also pointed out the scope of current materials research in India.

C.N.R. Rao, founder-president of MRS-I and director, Indian Institute of Science, Bangalore, delivered the MRS-I honor lecture entitled "Three Decades of Research in the Chemistry of MaterialsReflections on Three Recent Exciting Areas." He spoke about his intense involvement with research on oxides, including high-temperature superconductors, clusters, and fullerenes. Rao also was presented with the first MRS-I/ICSC Prize for his contributions to superconductivity and materials science.

In addition, there were three special lectures. Kiyotaka Wasa, Katsuchita Electric Companies, Japan, an honorary member of MRS-I, spoke on sputter deposition technology from a materials engineering perspective. M.A. Subramanian, Du Pont, United States, delivered a lecture on designing zeolites as novel chemical precursors to aluminosilicate-based ceramics. Tu Hailing, Beijing General Research Institute for Nonferrous Metals, China, presented a broad picture of the research on semiconducting materials at his center.

P. Chaddah (CAT, Indore) and A.K. Gupta (NPL, New Delhi) received the annual MRS-I/ICSC Superconductivity Awards. Sixteen MRS-I Medal awards were announced, with fourteen of the recipients also presenting lectures (see

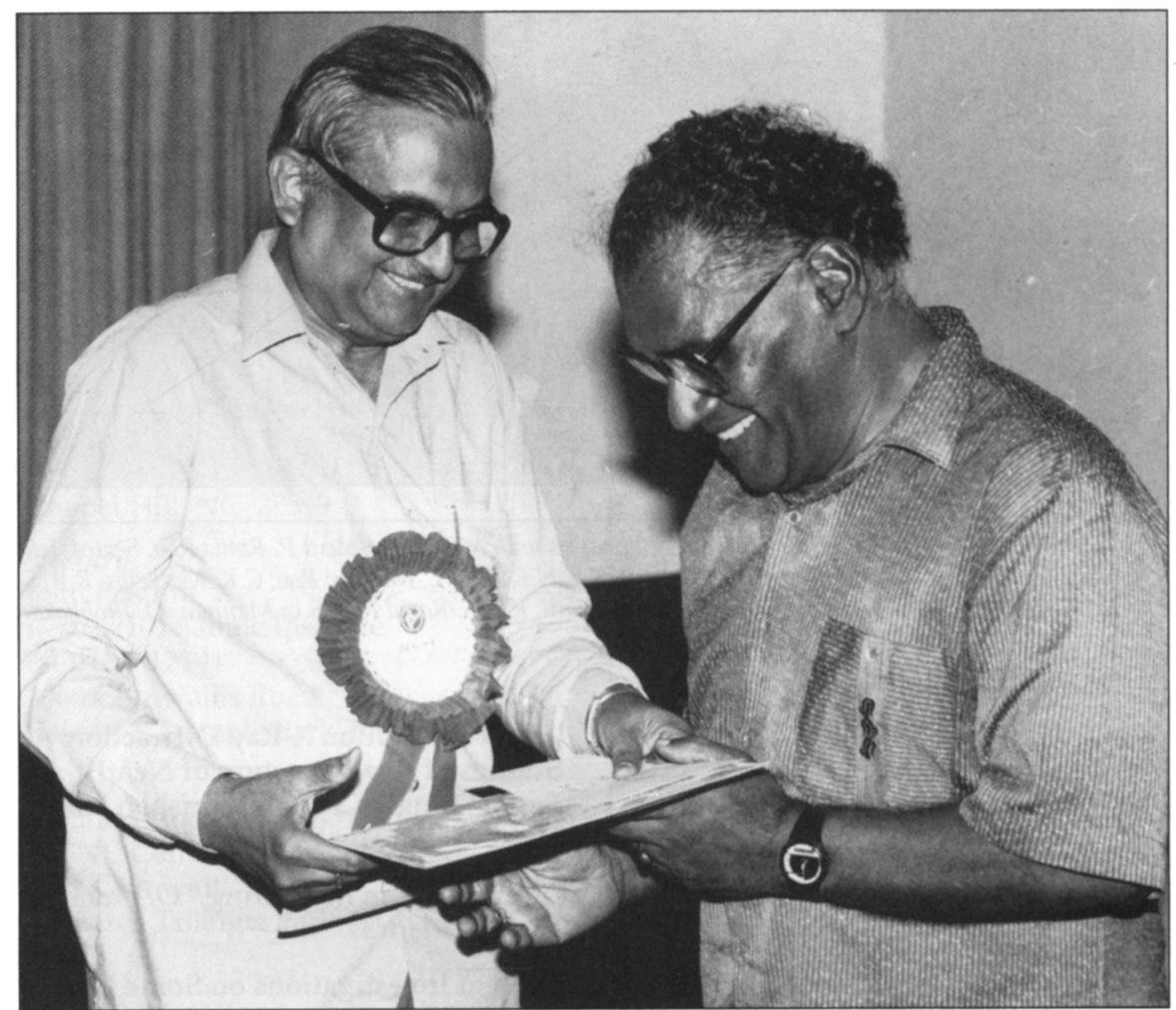

Figure 1. MRS-I president P. Rama Rao (left) presents the Distinguished Materials Scientist of the Year Award to C.N.R. Rao.

following list of winners and lectures). An award also was presented to $R$. Krishna Kumar, Department of Mechanical Engineering, Indian Institute of Technology, Madras, for authoring the paper "Dynamic Fracture Mechanics-A Scientific Tool for the Prevention of Catastrophic Failure." This work was judged as the best paper published in the Bulletin of Materials Science during 1992 (Vol. 15, February 1992, p. 55).

The poster session, which attracted lively participation, displayed 100 poster papers covering metals and alloys; polymers; thin-film synthesis, structure, and properties; electronic materials and devices; biomaterials; composites; ceramics and glasses; and superconductivity. Three posters were selected for "best poster" prizes.

S. Ranganathan General Secretary, MRS-I
MRS-I Medal Lectures 1993 "Sol-Gel Processing-A Versatile Concept for Special Glasses and Ceramics," D. Ganguli, CGCRI/CSIR, Calcutta.

"Development of Novel Sintered Cutting Tool Materials," G.S. Upadhyaya, IIT, Kanpur.

"Advanced Ceramics-Combustion Synthesis and Properties," K.C. Patil, IISc., Bangalore.

"Probing Exotic Magnetic Phases in Ferrites with Neutrons," L. Madhav Rao, BARC, Bombay.

"Sol-Gel Microsphere Pelletisation-A Powderfree and Economic Process for Fabrication of Ceramic Nuclear Fuel Pellets," C. Ganguly, BARC, Bombay.

"Some Recent Innovations in the Kroll Process of Titanium Sponge Production," R.B. Subramaniam, DMRL, Hyderabad. 


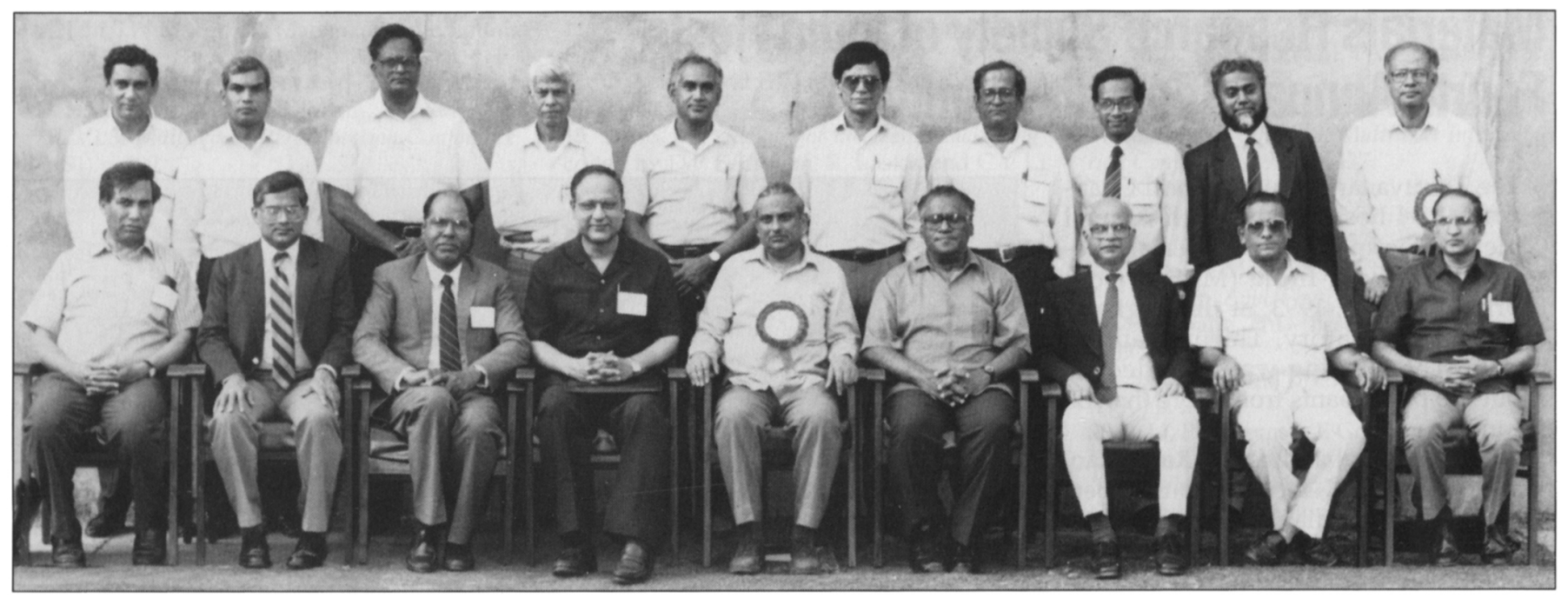

Figure 2. MRS-I Medal winners and other honorees with MRS-I president P. Rama Rao. Seated, left to right: Krishan Lal, Sanak Mishra, G.S. Upadhyaya, A.K. Chatteriee, P. Rama Rao, C.N.R. Rao, L. Madhav Rao, C.K. Mathews, R.B. Subramaniam. Standing, left to right: P. Chaddha, A.K. Gupta, K.A. Natarajan, K.C. Patil, K.V.S. Rama Rao, S.L. Mannan, O. Prabhakar, D. Ganguli, C. Ganguly, S. Ranganathan.

"Liquid Sodium-The Heat Transport Medium in Fast Breeder Reactors," C.K. Mathews, IGCAR, Kalpakkam.

"Race of New Inorganic Materials Towards an Ultra Neolithic Age," A.K. Chatterjee, ACC Ltd., Bombay.

"Towards Understanding of HighTemperature Deformation and Fracture," S.L. Mannan, IGCAR, Kalpakkam.
"High Resolution X-Ray Diffraction Studies of Real Structure of Nearly Perfect Single Crystals," Krishan Lal, NPL/CSIR, New Delhi.

"Solidification Modelling," O. Prabhakar, IIT, Madras.

"Recent Investigations on Some Cubic Laves' Phase Compounds of Rare Earth and Transition Metals," K.V.S. Rama Rao, IIT, Madras.
"The Unique Features of Textures in Low Carbon Steels," Sanak Mishra, SAIL R\&D, Ranchi.

"Biotechnology of Gold Processing," K.A. Natarajan, IISc., Bangalore.

The additional MRS-I medal winners were S.K. Date, NCL, Pune, and A.K. Shukla, IISc, Bangalore.

\title{
MRS Membership Special Introductory Offer still available-
}

\author{
July thru December: only \$30 \\ For fulltime students: only $\$ 10$
}

Call (412) 367-3003; Fax (412) 367-4373 